\title{
Decomposição de fontes orgânicas e mineralização de formas de nitrogênio e fósforo ${ }^{1}$
}

\author{
Eddi Alejandro Vanega Chacón ${ }^{2}$, Eduardo de Sá Mendonça $a^{3}$, Rubens Ribeiro da Silva $a^{4}$ Paulo César de Lima \\ Ivo Ribeiro da Silva ${ }^{6}$ e Reinaldo Bertola Cantarutti ${ }^{6}$
}

\section{RESUMO}

Diferentes resíduos in natura (farinhas, tortas e vinhaças) e compostos (lixo domiciliar e lodo de esgoto) foram avaliados quanto à decomposição e mineralização de formas orgânicas de N e P, quando incorporados a um Latossolo Vermelho-Amarelo, em condições de ambiente controlado. A decomposição foi determinada em experimento de respirometria e a mineralização, por incubação. Para um período de incubação de 30 dias, os resíduos in natura apresentaram maior evolução acumulada de $\mathrm{C}-\mathrm{CO}_{2}$ em comparação aos resíduos na forma compostada. No período de incubação de 60 dias, os compostos de lodo de esgoto (4.500 $\mathrm{mg} \mathrm{kg}^{-1}$ ) e lixo domiciliar $\left(2.000 \mathrm{mg} \mathrm{kg}^{-1}\right)$ mineralizaram N, em contraste com a farinha de carne e osso $\left(385 \mathrm{mg} \mathrm{kg}^{-1}\right)$ e a torta de filtro $\left(281 \mathrm{mg} \mathrm{kg}^{-1}\right)$, que imobilizaram $\mathrm{N}$. A relação $\mathrm{C} / \mathrm{N}$ e o teor inicial de $\mathrm{N}$ nos resíduos não explicaram o seu processo de mineralização. Isso sugeriu que a natureza do resíduo e características como formas de $\mathrm{C}$ facilmente biodegradáveis, teores e capacidade dos polifenóis totais solúveis em complexar proteína e, estoques de $\mathrm{N}$ nas formas fúlvica e húmica da matéria orgânica interferiram na mineralização de N. Para um período de incubação de 45 dias, a mineralização do P correlacionou-se positivamente com o teor inicial de $\mathrm{P}$ orgânico $(\mathrm{r}=0,99 * *)$ e negativamente com a relação $\mathrm{C} / \mathrm{P}\left(\mathrm{r}=-0,88^{* *}\right)$ dos resíduos. A farinha de carne e osso apresentou maior mineralização de $\mathrm{P}\left(1.144 \mathrm{mg} \mathrm{kg}^{-1}\right)$ em relação aos outros resíduos.

Palavras-chave: Resíduos industriais, compostos de lixo domiciliar e lodo de esgoto, respiração do solo.

\section{ABSTRACT}

\section{Decomposition of organic sources and mineralization of nitrogen and phosphorus forms}

Different raw residues (meals, cakes and distillery yeasts) or composts (sewage sludge and domestic waste) were evaluated for decomposition and mineralization of $\mathrm{N}$ and $\mathrm{P}$ organic forms. The materials were incorporated to soil samples from an B horizon of an Oxisol, without acidity correction, under controlled environmental conditions. Decomposition was determined by a respirometry experiment and mineralization by incubation. For an incubation period of 30 days, raw residues produced more accumulative quantities of $\mathrm{C}-\mathrm{CO}_{2}$ compared with composts. For an incubation period of 60 days, sewage sludge compost (4500 mg kg-1) and domestic waste $\left(2000 \mathrm{mg} \mathrm{kg}^{-1}\right)$ mineralized N, whereas meat and bone meal ( $\left.385 \mathrm{mg} \mathrm{kg}^{-1}\right)$ and filter cake $\left(281 \mathrm{mg} \mathrm{kg}^{-1}\right)$ immobilized it. The $\mathrm{C} / \mathrm{N}$ ratio and initial $\mathrm{N}$ content did not explain the pathways of $\mathrm{N}$ mineralization. This suggested that the nature of the residues and characteristics such as fraction of easily-biodegradable $\mathrm{C}$, total soluble polyphenol content and capacity to complex protein, as well

\footnotetext{
Recebido para publicação em janeiro de 2008 e aprovado em dezembrode 2010

1 Parte da tese apresentada à Universidade Federal de Viçosa, pelo primeiro autor, para obtenção do título de Doutor em Solos Nutrição de Plantas.

2 Engenheiro-Agrônomo, Doutor. Facultad de Agronomia, Universidad de San Carlos de Guatemala. Ciudad Universitaria, Zona 12, Ciudad de Guatemala, Guatemala.

3 Engenheiro-Agrônomo, PhD. Departamento de Produção Vegetal, Universidade Federal do Espírito Santo, Alto universitário s/n, Centro, 29500-000, Alegre, ES, Brasil. esmjplia@gmail.com.

4 Engenheiro-Agrônomo, Doutor. Departamento de Produção, Universidade Federal do Tocantins, C. Postal, 66, 774.000-000, Gurupi, TO, Brasil. rrs2002@uft.edu.br.

5 Engenheiro-Agrônomo, Doutor. Empresa de Pesquisa Agropecuária de Minas Gerais, Centro de Tecnologias da Zona da Mata, Vila Gianette, casa 46, 36570-000, Viçosa, MG, Brasil.

6 Engenheiro-Agrônomo, PhD. Departamento de Solos, Universidade Federal de Viçosa, Av. Peter Henry Rolfs s/n, Centro, 36570-000, Viçosa, MG, ivsilva@ ufv.br ;cantarutti@ufv.br.
} 
as the $\mathrm{N}$ pools in fulvic and humic acids of the organic matter were related with $\mathrm{N}$ mineralization. For an incubation period of 45 days, $\mathrm{P}$ mineralization was positively correlated with the initial organic $\mathrm{P}$ content $\left(\mathrm{r}=0,99^{* *}\right)$, and $\mathrm{C} / \mathrm{P}$ ratio $\left(\mathrm{r}=-0,88^{* *}\right)$ of the residues. Meat and bone meal showed the greatest content of mineralized $\mathrm{P}\left(1144 \mathrm{mg} \mathrm{kg}^{-1}\right)$.

Key words: Compost of sewage sludge and domestic waste, industrial residues, soil respiration.

\section{INTRODUÇÃO}

A produção de resíduos orgânicos está estreitamente relacionada com o crescimento populacional e industrial em todo o mundo. Existe preocupação quanto ao destino final desses resíduos para minimizar a poluição ambiental. Entre as várias formas de destinação, podem se mencionar a incineração, peletização e produção de energia. No entanto, a reciclagem via utilização agronômica, em suas diversas possibilidades (adubação de cultivos, recuperação de áreas degradadas, reflorestamento e compostagem), tem apresentado maior potencial devido à melhoria das características químicas, físicas e biológicas dos solos, diminuindo custos no processo produtivo (Melo \& Marques, 2000).

Aplicados raramente in natura, esses resíduos são incorporados ao solo em forma de composto, aportando grandes quantidades de substâncias húmicas. Pesquisas têm revelado que a adição de compostos de lodo de esgoto (biossólidos) tem aumentado a agregação do solo (Jorge et al., 1991); e a aplicação de compostos de lixo domiciliar, reduzido a resistência à penetração (Aggelides \& Londra, 2000), melhorando a qualidade física do solo.

Para a utilização agrícola faz-se necessário conhecer não apenas as características dos resíduos, mas também as do solo, da planta e do clima da localidade onde serão aplicados. Sabe-se, por exemplo, da presença de patógenos e parasitas no lodo de esgoto, bem como de metais pesados em resíduos provenientes de áreas com alta concentração de indústrias poluentes (Xin et al., 1992). Sua utilização como insumo agrícola deve estar condicionada ao monitoramento de sua qualidade química e microbiológica, teor inicial de metais pesados no solo, à quantidade total adicionada de metais pesados e toxidez desses elementos para as plantas (Kabata \& Pendias, 2000).

Os resíduos industriais têm sido utilizados com êxito em plantações florestais, principalmente em regiões de clima temperado (Gonçalves et al., 2000). Nos países tropicais, seu uso ainda é restrito e pouco estudado em condições de campo por maiores períodos de tempo, porém existem algumas experiências no seu emprego tanto na área agrícola (Oliveira et al., 1995) como na florestal (Vaz \& Gonçalves, 2002; Rocha et al., 2004).
Reconhecendo sua potencialidade como alternativa na manutenção de níveis adequados de matéria orgânica no solo, o uso eficiente dos resíduos derivados de atividades antrópicas deve se iniciar mediante o conhecimento das suas características químicas e bioquímicas no estado in natura, assim como do grau de estabilidade e maturação quando utilizados na forma de composto. Assim, considerando que o tipo de resíduo industrial interfere na dinâmica de decomposição e mineralização, o presente trabalho teve como objetivos: caracterizar química e bioquimicamente resíduos, in natura e na forma de composto, e avaliar a decomposição e mineralização de formas orgânicas de $\mathrm{N}$ e $\mathrm{P}$ após a incorporação ao solo em ambiente controlado.

\section{MATERIAL E MÉTODOS}

O presente trabalho foi conduzido no Departamento de Solos da Universidade Federal de Viçosa (UFV). Para a realização do trabalho foram selecionadas oito fontes orgânicas in natura e na forma de composto, em municípios dos Estados de Minas Gerais e São Paulo. As fontes orgânicas selecionadas foram: farinha de osso; farinha de carne e osso; torta de mamona; torta de filtro; vinhaça de cachaça; vinhaça de álcool; composto de lodo de esgoto; e composto de lixo. Em cada fonte orgânica foi realizada uma amostragem composta resultante de 10 amostras simples $\left(50 \mathrm{~cm}^{3}\right)$, segundo a resolução CONAMA 375/07.

\section{Caracterização química}

Para a caracterização química, três subamostras de cada fonte orgânica foram secas em estufa com circulação forçada de ar a $65^{\circ} \mathrm{C}$ durante 72 horas, moídas e passadas em peneira com malha de $2 \mathrm{~mm}$. A caracterização realizada incluiu os atributos: teor de matéria orgânica total, pelo método da perda por ignição (Kiehl, 1985); teor de C orgânico total, por oxidação úmida com aquecimento externo (Yeomans \& Bremner 1988); N total, pelo método Kjeldahl; elementos totais após digestão nítrico-perclórica, determinando-se nos extratos os teores de $\mathrm{S}, \mathrm{Al}, \mathrm{Fe}, \mathrm{Mn}, \mathrm{Cu}$, $\mathrm{Cr}, \mathrm{Cd}, \mathrm{Pb}$ e $\mathrm{Zn}$ por espectrometria de emissão de plasma; $\mathrm{Ca}$ e Mg, por espectrofotometria de absorção atômica; K, por espectrometria de emissão de chama; e P, por colorimetria (Murphy \& Riley, 1962). 
Com base nos resultados de $\mathrm{C}$ e macronutrientes, foram formados grupos diferentes para as fontes orgânicas, utilizando-se análise multivariada, com a técnica de componentes principais (Ribeiro Junior, 2001). Após o agrupamento, foram selecionadas fontes orgânicas representativas de cada grupo para caracterização bioquímica e realização de ensaios de decomposição e mineralização de formas orgânicas de $\mathrm{N}$ e $\mathrm{P}$.

\section{Caracterização bioquímica}

A caracterização bioquímica consistiu da determinação do C solúvel em água, por colorimetria, utilizando-se Mn(III)-pirofosfato em meio ácido como agente oxidante (Bartlett \& Ross, 1988); carboidratos totais solúveis, pelo método da antrona (Brink et al., 1960); polifenóis totais solúveis, extraídos com metanol (50\%) e determinados por colorimetria, utilizando-se o reagente de Folin-Denis; e a capacidade dos polifenóis em complexar proteína, pelo método Blue-Dye Labelled Bovine Serum Albumin (BSA), (Anderson \& Ingram, 1989). Adicionalmente, para as fontes na forma de composto foi feito o fracionamento das substâncias húmicas por solubilidade diferencial, utilizando-se os conceitos de frações húmicas estabelecidos pela Sociedade Internacional de Substâncias Húmicas, descritos por Hayes et al. (1989). Nessas frações foram determinados os teores de C (Yeomans \& Bremner, 1988); N total, pelo método Kjeldahl; e o índice de humificação pela equação:

$$
I H=\left(\frac{F A F+F A H}{C \text { total }}\right) .100
$$

em que:

IH = índice de humificação;

FAF = fração ácidos fúlvicos;

$\mathrm{FAH}=$ fração ácidos húmicos; e

C total $=$ carbono orgânico total.

\section{Decomposição}

Nos ensaios de decomposição e mineralização foram utilizadas amostras do horizonte B de um Latossolo Vermelho-Amarelo distrófico, de textura argilosa (TFSA), sem correção da acidez. A caracterização química constou de: pH em água de 4,57; P de 0,6 $\mathrm{mg} \mathrm{dm}^{-3}$; $\mathrm{K}_{\text {de }} 6 \mathrm{mg} \mathrm{dm}^{-3}$; $\mathrm{H}+\mathrm{Al}$ de 5,9 $\mathrm{cmol}_{\mathrm{c}} \mathrm{dm}^{-3}$; soma de bases trocáveis (SB) de $0,04 \mathrm{cmol}_{\mathrm{c}} \mathrm{dm}^{-3}$; capacidade de troca catiônica (CTC) de $5,94 \mathrm{cmol}_{\mathrm{c}} \mathrm{dm}^{-3}$; capacidade máxima de adsorção de $\mathrm{P}$ (CMAP) de 1,32 $\mathrm{mg} \mathrm{g}^{-1}$; e teor de matéria orgânica de 3,08 dag $\mathrm{kg}^{-1}$.

Os experimentos foram conduzidos seguindo delineamento experimental inteiramente casualizado, com quatro repetições. Foi adotado o esquema de parcelas subdivididas em função do tempo, no qual as fontes orgânicas corresponderam às parcelas e as avaliações sucessivas de $\mathrm{C}-\mathrm{CO}_{2}, \mathrm{~N}$ e $\mathrm{P}$, às subparcelas. As quantificações de emissões de $\mathrm{C}-\mathrm{CO}_{2}$ realizadas a cada dois dias (sete determinações) inicialmente e a cada três dias (cinco determinações) posteriormente e as avaliações da mineralização de formas orgânicas de $\mathrm{N}$ e $\mathrm{P}$ foram realizadas durante oito e seis semanas, respectivamente.

Cada unidade experimental foi composta da massa equivalente a $2 \mathrm{~g}$ de $\mathrm{C}$ de cada fonte orgânica misturada a $100 \mathrm{~g}$ de solo seco (Paul \& Clark, 1996) acondicionados em potes plásticos cilíndricos, com $15 \mathrm{~cm}$ de altura e $10 \mathrm{~cm}$ de diâmetro (unidade experimental). Como testemunha foi mantida unidade experimental apenas com solo para fins de comparação. As unidades experimentais foram mantidas a uma umidade equivalente a $80 \%$ da capacidade de campo e em câmara de incubação a $25 \pm 1^{\circ} \mathrm{C}$.

\section{Mineralização de C}

$\mathrm{O}$ C- $\mathrm{CO}_{2}$ respirado foi capturado em solução de $\mathrm{NaOH}$ $0,5 \mathrm{~mol} \mathrm{~L}{ }^{-1}$, formando carbonato com a adição de $\mathrm{BaCl}_{2}$ $0,05 \mathrm{~mol} \mathrm{~L}^{-1}$ e dosado por meio de titulação com $\mathrm{HCl} 0,25$ mol L-1. A massa de C- $\mathrm{CO}_{2}$ evoluída foi expressa em termos absolutos (Anderson, 1982) e a cinética de decomposição, ajustada pelo modelo de saturação de Hill (Morgan et al., 1975):

$$
Y=\frac{\alpha^{*} X^{\delta}}{\gamma+X^{\delta}}+\varepsilon
$$

em que:

$\alpha=$ parâmetro relativo à assíntota, que caracteriza a saturação;

$\gamma=$ parâmetro de crescimento da função, chamado também de constante do processo;

$\delta=$ ordem aparente do processo;

$\chi=$ tempo; $\mathrm{e}$

$\varepsilon=$ erro aleatório.

\section{Mineralização de $N$}

Em amostras coletadas semanalmente, foram determinados o $\mathrm{N}$ amoniacal $\left(\mathrm{N}-\mathrm{NH}_{4}^{+}\right)$e o nítrico $\left(\mathrm{N}-\mathrm{NO}_{3}^{-}\right)$, extraídos com $\mathrm{KCl} 1 \mathrm{~mol} \mathrm{~L}^{-1}$ e determinados por colorimetria segundo Kempers \& Zweers (1986) e Yang et al. (1998), respectivamente. $\mathrm{O} \mathrm{N}$ inorgânico $(\mathrm{Ni})$ foi calculado como a soma do $\mathrm{N}_{-} \mathrm{NH}_{4}{ }^{+}+\mathrm{N}_{-} \mathrm{NO}_{3}{ }^{-}$. Aos teores observados acumulados de $\mathrm{N}$ inorgânico foi ajustado um modelo exponencial simples:

$$
\mathrm{Ni}=\mathrm{A} \mathrm{e}^{\mathrm{kt}}
$$

em que:

$\mathrm{Ni}=$ teor de $\mathrm{N}$ inorgânico acumulado; 
$\mathrm{A}=$ teor de $\mathrm{N}$ inorgânico proveniente do compartimento facilmente mineralizável;

$\mathrm{k}=$ taxa constante de mineralização; $\mathrm{e}$

$\mathrm{t}=$ tempo

\section{Mineralização de $P$}

As determinações do $\mathrm{P}$ inorgânico $(\mathrm{Pi})$ e do $\mathrm{P}$ total $(\mathrm{Pt})$ foram realizadas mediante fracionamento químico das formas lábeis (extração com água, resina de troca aniônica e bicarbonato $0,5 \mathrm{~mol} \mathrm{~L}^{-1}$ ), pelo método de Hedley et al. (1982) modificado. O P orgânico (Po) foi obtido pela diferença entre $\mathrm{Pt}$ e $\mathrm{Pi}$. O modelo linear simples foi ajustado aos dados observados acumulados de Pi.

Os dados qualitativos foram submetidos a análise de variância, teste de médias (Tukey, 0,05) e os quantitativos a análise de regressão utilizando-se o programa SAEG.

De acordo com suas características os resíduos foram agrupados empregando-se a Análise de Componentes Principais (ACP) (SAS, 1998).

\section{RESULTADOS E DISCUSSÃO}

As fontes orgânicas mostraram grande variabilidade em suas características químicas (Tabela 1). Os teores médios de C orgânico foram da ordem de 20 dag $\mathrm{kg}^{-1}$. A farinha de carne e osso e a torta de mamona apresentaram os maiores teores de $\mathrm{N}$ e $\mathrm{Mg}$; a farinha de osso e a de carne e osso apresentaram os maiores teores de $\mathrm{P}$ e $\mathrm{Ca}$, com relação $\mathrm{Ca} / \mathrm{P}$ de aproximadamente 2; o lodo de esgoto apresentou o maior teor de $\mathrm{S}$; e a torta de mamona, maior de K. As fontes orgânicas estudadas apresentaram relação $\mathrm{C} / \mathrm{N}<20$; relação $\mathrm{C} / \mathrm{P}<200$ e relação $\mathrm{C} / \mathrm{S} \leq 200$, indicando a potencialidade de mineralização no curto prazo (Stevenson \& Cole, 1999). Os compostos de lodo de esgoto e lixo domiciliar apresentaram teores intermediários de $\mathrm{Fe}$, $\mathrm{Cu}$ e $\mathrm{Zn}$, embora com os maiores teores de $\mathrm{Cr}$, $\mathrm{Cd}$ e $\mathrm{Pb}$. Apesar disso, os compostos apresentaram teores de metais pesados menores que os estabelecidos como referência pela CETESB (1999). Segundo a legislação do Ministério da Agricultura, para adubos orgânicos simples e compostos (Brasil, 2004) o teor de matéria orgânica e de $\mathrm{N}$ total e a relação $\mathrm{C} / \mathrm{N}$ dos resíduos estão dentro das especificações, mas são resíduos (> 25\%) e compostos (> $40 \%$ ) com alto teor de umidade, exceto farinha de osso, lixo domiciliar e torta de mamona.

Os grupos formados mediante técnica de componentes principais foram: grupo 1 , farinha de osso e farinha de carne e osso; grupo 2, torta de mamona, torta de filtro e composto de lodo de esgoto; e grupo 3, composto de lixo domiciliar, vinhaça de cachaça e vinhaça de álcool (Figura 1).

A torta de filtro destacou-se das outras fontes orgânicas por apresentar maior teor de C solúvel em água, carboidratos e polifenóis totais solúveis com maior capacidade de complexar proteína (Tabela 2). A farinha de carne e osso também apresentou uma fração rica em formas de C facilmente biodegradáveis, não obstante sua matriz orgânica ser constituída de materiais graxos (Kiehl, 1985), com menor teor de polifenóis totais solúveis, mas com capacidade intermediária de complexar proteína. Ambos os resíduos mostraram potencial de imobilização de N no curto prazo (Freitas et al., 1988; Tedesco et al., 1999). Os compostos de lixo domiciliar e lodo de esgoto apresentaram os menores teores de C solúvel em água e carboidratos, produto de sua estabilidade, com teores intermediários de polifenóis, porém com menor capacidade de complexar proteína; ambos os compostos mostraram potencial de mineralização de N no curto prazo (Kiehl, 2005).

O composto de lixo domiciliar, quando comparado com o de lodo de esgoto, apresentou menor teor de C orgânico total, maior teor de $\mathrm{C}$ na fração ácidos fúlvicos, teor similar na fração ácido húmico e aproximadamente a metade daquele da fração humina no lodo de esgoto, apresentando maior índice de humificação (Tabela 3). O lodo de esgoto apresentou maiores teores de $\mathrm{N}$ total e $\mathrm{N}$ nas frações ácidos húmicos, fúlvicos e humina, quando comparado com o lixo domiciliar. Apesar disso, ambos os compostos tiveram igual relação das frações ácidos húmicos/ ácidos fúlvicos, sugerindo igual magnitude nas formas orgânicas de $\mathrm{N}$ facilmente biodegradáveis. Contudo, o lixo domiciliar apresentou relação $\mathrm{C} / \mathrm{N}$ na fração ácidos fúlvicos de 14 e o lodo de esgoto de 3 , sugerindo maior potencial de mineralização de $\mathrm{N}$ da fração facilmente biodegradável para o lodo de esgoto. $\mathrm{Na}$ fração ácido húmico, o lixo domiciliar também mostrou maior relação $\mathrm{C} /$ $\mathrm{N}$ em comparação com o lodo de esgoto.

\section{Decomposição}

Houve variação entre as quantidades evoluídas de C$\mathrm{CO}_{2}$; os tratamentos com a farinha de carne e osso e a torta de filtro apresentaram as maiores quantidades de $\mathrm{C}$ em forma facilmente biodegradáveis (Figura 2). A matriz orgânica da farinha de carne e osso (34\% de sua constituição) é composta de aproximadamente $7 \%$ de matéria graxa e $27 \%$ de osseína com 5\% de N (Kiehl, 1985), com teores de aminoácidos proporcionais aos de proteína bruta (Rostagno et al., 2000), apresentando uma fração de fácil biodegradação no curto prazo; não obstante, caracteriza-se por ser um material de difícil decomposição ao longo do tempo (Malavolta, 1981). Por essa razão, são sugeridas técnicas de desengorduração (Tedesco et al., 1999) e pirólise (Deydier, 2005) para facilitar sua decomposição e incrementar os lucros na sua comercialização como adubo de fácil decomposição.

Considerando uma eficiência de assimilação de $\mathrm{C}$ pelos microrganismos de 40\% (Siqueira, 1988), estimou-se que en- 


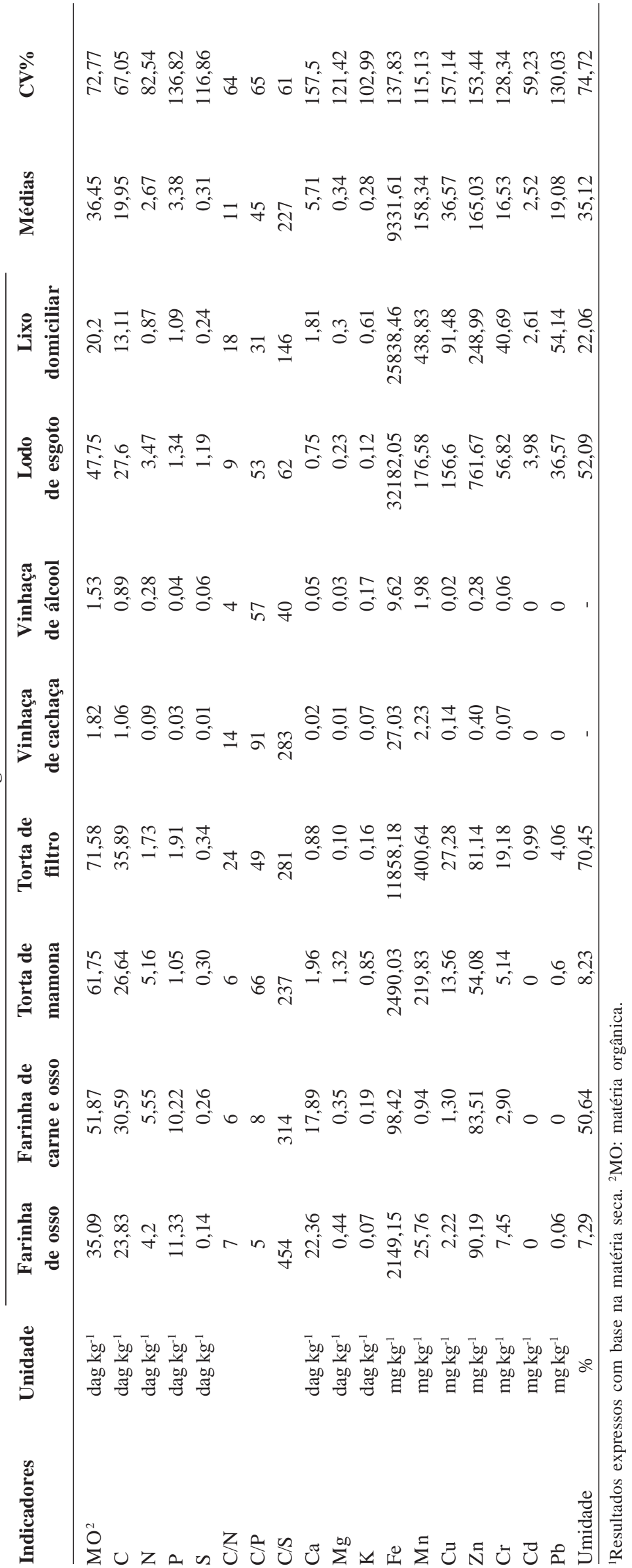




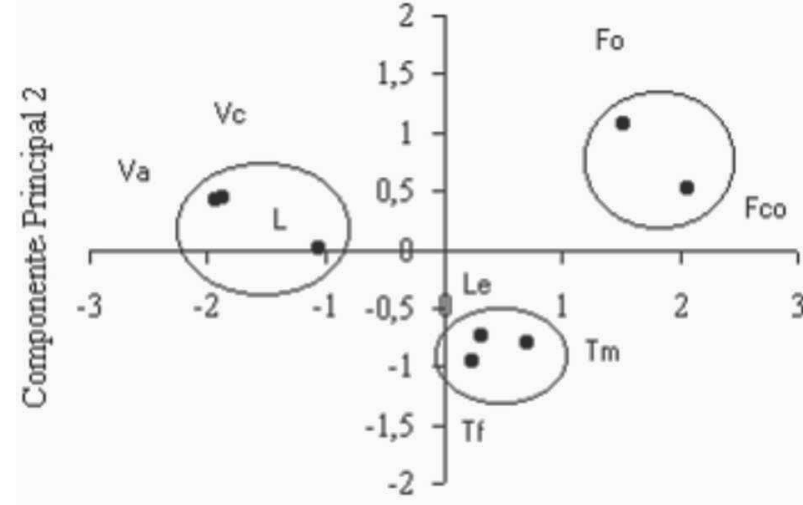

\section{Componente Principal 1}

Figura 1. Dispersão de escores e agrupamento por similaridade para os diferentes resíduos. Fo: farinha de osso; Fco: farinha de carne e osso; Le: lodo de esgoto; Tm: torta de mamona; Tf: torta de filtro; L: composto de lixo domiciliar; Vc: vinhaça de cachaça; e Va: vinhaça de álcool.

tre 2,57 e 27,59\% do $\mathrm{C}$ adicionado por meio dos diferentes tratamentos foi oxidado durante o processo de decomposição (Tabela 4). Os tratamentos com compostos foram os que apresentaram as menores proporções de $\mathrm{C}$ oxidado, devido à presença de formas mais estáveis do C (Kiehl, 2005).

A produção acumulada de $\mathrm{C}-\mathrm{CO}_{2}$ evoluído ajustou-se adequadamente ao modelo de saturação de Hill (Tabela 5). Os maiores valores para a saturação na evolução de $\mathrm{C}-\mathrm{CO}_{2}$ (parâmetro $\alpha$ ) foram estimados para a fonte farinha de carne e osso, seguido pela fonte torta de filtro, indicando que esses resíduos apresentaram maior proporção de $\mathrm{C}$ facilmente oxidável. Houve grande variação $(\mathrm{CV}=106 \%)$ entre as esti- mativas da constante de evolução de $\mathrm{C}-\mathrm{CO}_{2}$ (parâmetro $\gamma$ ), sugerindo que essa foi sensível às variações qualitativas entre os resíduos. A cinética de evolução de $\mathrm{C}-\mathrm{CO}_{2}$ mostrou diferentes padrões de crescimento e variação $(\mathrm{CV}=27,37 \%)$ na ordem aparente do processo de saturação (parâmetro $\delta$ ).

A estimativa da taxa máxima de evolução de $\mathrm{C}-\mathrm{CO}_{2}$ correlacionou-se positivamente com os valores observados acumulados da evolução de $\mathrm{C}-\mathrm{CO}_{2}(\mathrm{r}=0,93 * *)$, sugerindo sua utilização como indicador da decomposição dos resíduos estudados. A taxa máxima de evolução foi atingida entre os cinco e sete dias de incubação para os tratamentos com farinha de carne e osso, composto de lixo domiciliar e lodo de esgoto, corroborando a existência de uma fração de fácil degradação na farinha de carne e osso. Já o tratamento com torta de filtro precisou de 13 dias para atingir a taxa máxima. Ao final do período de incubação, os valores acumulados de C-CO $\mathrm{CO}_{2}$ correlacionaram-se com os teores de $\mathrm{C}$ solúvel em água $\left(\mathrm{r}=0,57^{* *}\right)$, carboidratos $\left(\mathrm{r}=0,69^{* *}\right)$ e relação $\mathrm{C} / \mathrm{N}(\mathrm{r}=$ - 0,56**) dos materiais estudados. Para estas fontes orgânicas não houve associação entre decomposição e teor de polifenóis solúveis, porque os resíduos in natura apresentaram menores teores de polifenóis, mas com maior capacidade em complexar proteína.

A incorporação dos resíduos ao solo apresentou dinâmica de mineralização de $\mathrm{N}$ diferente para cada material (Figura 3), estando esse fato relacionado à natureza dos resíduos, já que os compostos apresentaram formas estáveis do $\mathrm{C}$, baixa relação $\mathrm{C} / \mathrm{N}$ e teor de polifenóis totais solúveis com menor capacidade de complexar proteína em relação aos resíduos in natura.

Tabela 2. Caracterização bioquímica de diferentes materiais orgânicos

\begin{tabular}{lcccr}
\hline \multirow{2}{*}{ Material } & \multicolumn{1}{c}{ Cag } & POL & CARB & \multicolumn{1}{c}{ BSA } \\
\cline { 2 - 3 } & & $\%$ & & $\mu_{\mathrm{g} \mathrm{mg}}^{-1}$ \\
\hline Farinha de carne e osso & 1,02 & 0,25 & 0,39 & 24,09 \\
Composto de lixo & 0,34 & 0,37 & 0,084 & 6,68 \\
Torta de filtro & 1,25 & 0,82 & 0,42 & 38,44 \\
Composto de lodo de esgoto & 0,52 & 0,43 & 0,090 & 6,63 \\
\hline
\end{tabular}

Cag: carbono solúvel em água; POL: polifenóis totais solúveis; CARB: carboidratos totais solúveis; e BSA: capacidade dos polifenóis em complexar proteína.

Tabela 3. Teores de $\mathrm{C}$ e $\mathrm{N}$ total e frações húmicas extraídas dos compostos de lixo domiciliar e lodo de esgoto

\begin{tabular}{|c|c|c|c|c|c|c|}
\hline \multirow{2}{*}{ Material } & $\mathrm{C}$ & FAF & FAH & FH & IH & \multirow{2}{*}{ FAH/FAF } \\
\hline & dag $\mathrm{kg}^{-1}$ & & $\mathrm{~g} \mathrm{~kg}^{-1}$ & & $\%$ & \\
\hline Lixo domiciliar & 13,11 & 15,80 & 6,20 & 106,60 & 16,78 & 0,39 \\
\hline Lodo de esgoto & 27,60 & 10,30 & 5,60 & 255,80 & 5,76 & 0,54 \\
\hline \multirow{2}{*}{ Material } & $\mathbf{N}$ & FAF & FAH & FH & & \multirow{2}{*}{ FAH/FAF } \\
\hline & dag $\mathrm{kg}^{-1}$ & & $\mathrm{~g} \mathrm{~kg}^{-1}$ & & & \\
\hline Lixo domiciliar & 0,87 & 1,30 & 1,10 & 5,70 & & 0,85 \\
\hline Lodo de esgoto & 3,47 & 4,30 & 3,70 & 25,50 & & 0,86 \\
\hline
\end{tabular}

C: carbono total; N: nitrogênio total; FAF: fração ácidos fúlvicos; FAH: fração ácidos húmicos; FH: fração humina; FAH/FAF: relação das frações ácidos húmicos/ácidos fúlvicos; e IH: índice de humificação.

Rev. Ceres, Viçosa, v. 58, n.3, p. 373-383, mai/jun, 2011 


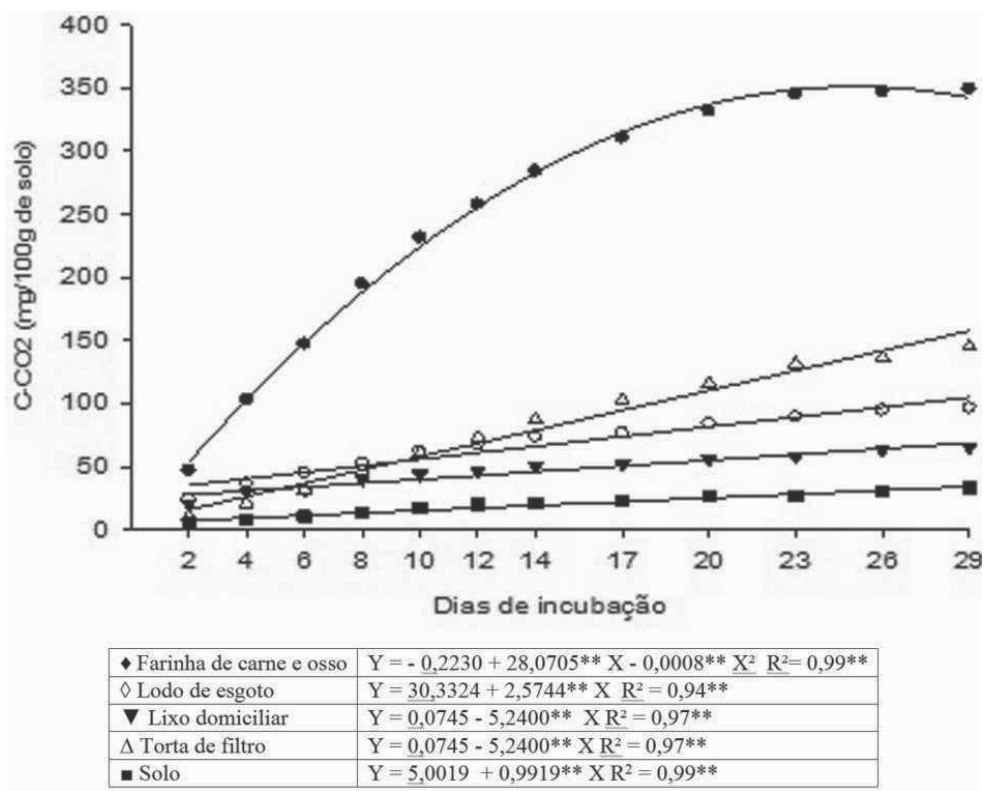

Figura 2. Produção acumulada de $\mathrm{C}-\mathrm{CO}_{2}$ do solo tratado com diferentes fontes orgânicas e testemunha (solo) durante o período de incubação.

Tabela 4. Evolução e oxidação do carbono do solo tratado com resíduos e testemunha (solo) durante o período de incubação

\begin{tabular}{|c|c|c|c|c|}
\hline \multirow{2}{*}{ Fontes orgânicas } & $\mathrm{C}-\mathrm{CO}_{2}$ & C & Cox & \multirow{2}{*}{$\begin{array}{r}\mathrm{C}-\mathrm{CO}_{2} \mathbf{o x} \\
\%\end{array}$} \\
\hline & & $\mathrm{mg}$ & & \\
\hline Farinha de carne e osso & 363,48 a & 242,32 & 605,8 & 27,59 \\
\hline Lixo domiciliar & $63,24 \mathrm{~d}$ & 42,16 & 105,4 & 2,57 \\
\hline Torta de filtro & $143,04 \mathrm{~b}$ & 95,36 & 238,4 & 9,22 \\
\hline Lodo de esgoto & $97,44 \mathrm{c}$ & 64,96 & 162,4 & 5,42 \\
\hline Solo & $32,40 \mathrm{e}$ & 21,6 & 54 & \\
\hline
\end{tabular}

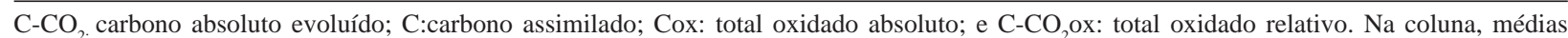
seguidas de mesma letra não diferem entre si pelo teste de Tukey $(\alpha=0,05)$.

Tabela 5. Estimadores dos parâmetros, intervalos de confiança assintóticos (IC): limite inferior (LI) e limite superior (LS), quadrado do coeficiente de correlação $\left(\mathrm{R}^{2}\right)$, dia em que ocorreu a taxa máxima de evolução e taxa máxima de evolução de $\mathrm{C}-\mathrm{CO}_{2}$, de conformidade com o modelo de Hill

\begin{tabular}{|c|c|c|c|c|c|c|}
\hline \multirow{2}{*}{ Tratamento } & \multirow{2}{*}{$\begin{array}{c}\text { Estimadores } \\
\text { dos parâmetros (1) }\end{array}$} & \multicolumn{2}{|c|}{ IC } & \multirow{2}{*}{$\mathbf{R}^{2}$} & \multirow{2}{*}{ Dias } & \multirow{2}{*}{$\begin{array}{c}\text { Taxa máxima de } \\
\mathrm{C}-\mathrm{CO}_{2} \mathrm{mg} \mathrm{d}^{-1}\end{array}$} \\
\hline & & $\mathbf{L}$ & $\mathbf{L S}$ & & & \\
\hline \multirow[t]{3}{*}{ Farinha de carne e osso } & $\alpha=445,10$ & 422,50 & 467,70 & 0,99 & 7 & 108,60 \\
\hline & $\gamma=22,61$ & 19,60 & 25,61 & & & \\
\hline & $\sigma=1,35$ & 1,26 & 1,45 & & & \\
\hline \multirow[t]{3}{*}{ Composto de lixo domiciliar } & $\alpha=90,00$ & 83,44 & 96,84 & 0,99 & 5 & 28,32 \\
\hline & $\gamma=7,29$ & 6,92 & 7,66 & & & \\
\hline & $\sigma=0,83$ & 0,77 & 0,89 & & & \\
\hline \multirow[t]{3}{*}{ Torta de filtro } & $\alpha=226,10$ & 193,60 & 258,60 & 0,99 & 13 & 77,32 \\
\hline & $\gamma=92,46$ & 73,68 & 111,30 & & & \\
\hline & $\sigma=1,50$ & 1,35 & 1,66 & & & \\
\hline \multirow[t]{3}{*}{ Composto de lodo de esgoto } & $\alpha=175,50$ & 140,60 & 210,40 & 0,99 & 5 & 37,56 \\
\hline & $\gamma=12,52$ & 11,02 & 14,03 & & & \\
\hline & $\sigma=0,81$ & 0,71 & 0,91 & & & \\
\hline \multirow[t]{3}{*}{ Solo (2) } & $\alpha=52,80$ & 46,21 & 59,39 & 0,99 & 3 & 4,00 \\
\hline & $\gamma=27,66$ & 25,19 & 30,13 & & & \\
\hline & $\sigma=1,12$ & 1,02 & 1,21 & & & \\
\hline
\end{tabular}

(1) Para todos os tratamentos, os estimadores dos parâmetros a, g e d foram significativos a $5 \%$ de probabilidade pelo teste de $\mathrm{t}$.

(2) Utilizou-se como fonte de carbono o carbono orgânico total do solo. 


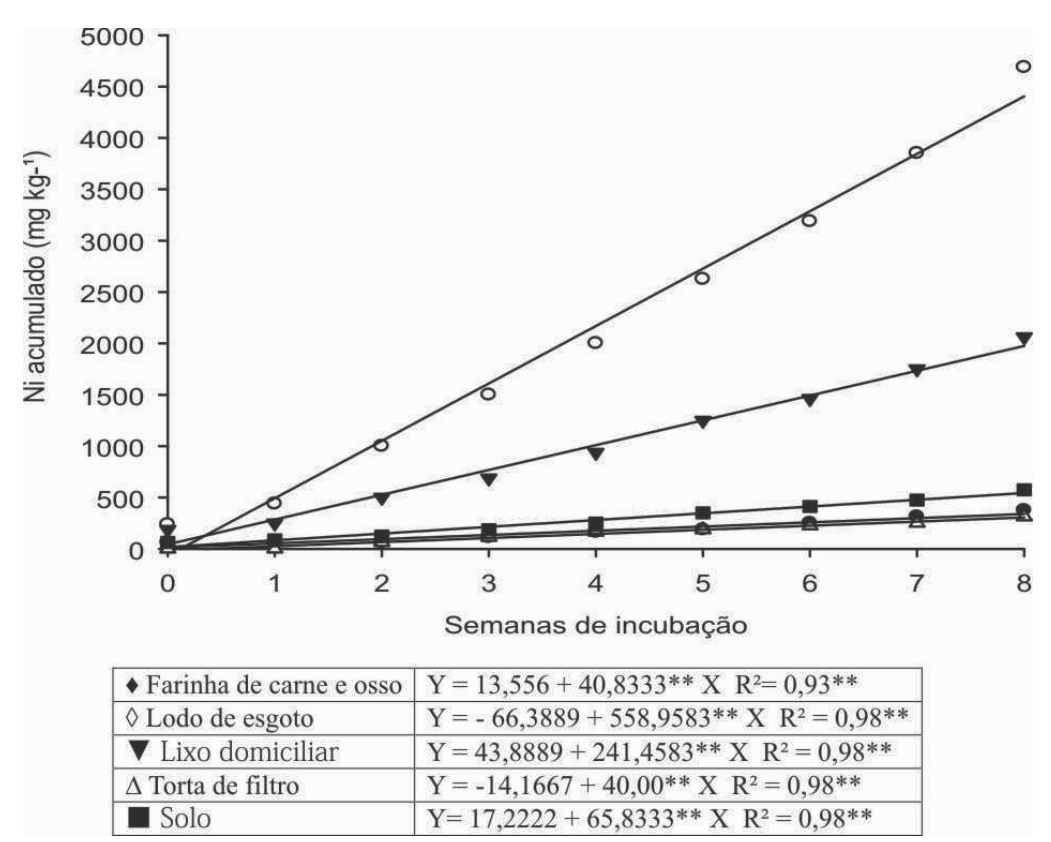

Figura 3. Teores acumulados de $\mathrm{N}$ inorgânico (Ni) para o solo tratado com resíduos e testemunha (solo) durante o período de incubação.

Os tratamentos com compostos apresentaram maiores teores de $\mathrm{N}$ inorgânico, provenientes do compartimento facilmente mineralizável (estimador do parâmetro A, Tabela 6). As taxas de mineralização de $\mathrm{N}$ dos tratamentos com compostos e torta de filtro foram da ordem de $0,20 \mathrm{mg} \mathrm{kg}$ por semana. $\mathrm{O}$ tratamento com a torta de filtro apresentou o menor teor acumulado de $\mathrm{N}$ inorgânico ao final do período de incubação. Isso se explica pela natureza dos compostos (Kiehl, 2005) e pelas características bioquímicas da cana-de- açúcar (Oliveira et al., 1999). Em relação à testemunha, o solo tratado com a farinha de carne e osso, mesmo acarretando imobilização de $\mathrm{N}$, apresentou taxa incipiente de mineralização $\left(0,32 \mathrm{mg} \mathrm{kg}^{-1}\right.$ por semana). Os teores acumulados de $\mathrm{N}$ inorgânico dos dife- rentes tratamentos correlacionaram-se negativamente com a capacidade dos polifenóis em complexar proteína $(\mathrm{r}=$ $\left.0,80^{* *}\right)$ dos resíduos. Não se observou associação entre os teores acumulados de $\mathrm{N}$ inorgânico e a relação $\mathrm{C} / \mathrm{N}$ dos resíduos.

Como a capacidade dos polifenóis em formar complexos resistentes com proteínas e enzimas fúngicas extracelulares é da mesma magnitude (BSA $\cong 7 \mu \mathrm{g} \mathrm{m}^{-1}$ ), a diferença nos teores de $\mathrm{N}$ mineralizado pode ser explicada pela menor relação $\mathrm{C} / \mathrm{N}$ na fração fúlvica e pelo maior teor de $\mathrm{N}$ total do lodo de esgoto.

$\mathrm{O}$ teor de $\mathrm{P}$ foi avaliado em pré-incubação (tempo 0 ) e após duas semanas de incubação. No tempo 0 , as formas predominantes do $\mathrm{P}$ foram as do $\mathrm{P}$ orgânico, com média

Tabela 6. Estimadores dos parâmetros, intervalos de confiança assintóticos (IC): limite inferior (LI) e limite superior (LS), quadrado do coeficiente de correlação $\left(\mathrm{R}^{2}\right)$ e teores observados acumulados de $\mathrm{N}$ inorgânico ao final do período de incubação para o solo tratado com diferentes resíduos e testemunha (solo)

\begin{tabular}{|c|c|c|c|c|c|}
\hline \multirow{2}{*}{ Tratamento } & \multirow{2}{*}{$\begin{array}{c}\text { Estimadores dos } \\
\text { parâmetros }^{1}\end{array}$} & \multicolumn{2}{|c|}{ IC } & \multirow{2}{*}{$\mathbf{R}^{2}$} & \multirow{2}{*}{$\begin{array}{c}\text { Ni acumulado } \\
\mathrm{mg} \mathrm{kg}^{-1}\end{array}$} \\
\hline & & U & $\mathbf{L S}$ & & \\
\hline \multirow[t]{2}{*}{ Farinha de carne e osso } & $A=31,21$ & 17,33 & 45,10 & 0,97 & $385 \mathrm{~d}$ \\
\hline & $\mathrm{k}=0,32$ & 0,25 & 0,38 & & \\
\hline \multirow[t]{2}{*}{ Composto de lixo domiciliar } & $A=310,90$ & 214,3 & 407,5 & 0,97 & $2.000 \mathrm{~b}$ \\
\hline & $\mathrm{k}=0,23$ & 0,19 & 0,28 & & \\
\hline \multirow[t]{2}{*}{ Torta de filtro } & $A=52,57$ & 36,49 & 69,65 & 0,95 & $281 \mathrm{e}$ \\
\hline & $\mathrm{k}=0,21$ & 0,16 & 0,26 & & \\
\hline \multirow[t]{2}{*}{ Composto de lodo de esgoto } & $\mathrm{A}=739,10$ & 555,40 & 922,80 & 0,98 & $4.500 \mathrm{a}$ \\
\hline & $\mathrm{k}=0,22$ & 0,19 & 0,26 & & \\
\hline \multirow[t]{2}{*}{ Solo } & $A=51,06$ & 28,96 & 73,17 & 0,97 & $535 \mathrm{c}$ \\
\hline & $\mathrm{k}=0,30$ & 0,23 & 0,36 & & \\
\hline
\end{tabular}

(1) Para todos os materiais, os estimadores dos parâmetros foram significativos a 5\% de probabilidade pelo teste de t. Na coluna, médias seguidas de pelo menos uma mesma letra não diferem entre si pelo teste de Tukey $(\mathrm{a}=0,05)$.

Rev. Ceres, Viçosa, v. 58, n.3, p. 373-383, mai/jun, 2011 
Tabela 7. Teores de P orgânico e inorgânico, antes e após duas semanas de incubação, e P inorgânico acumulado ao final do período de incubação para o solo tratado com diferentes resíduos e testemunha (solo)

\begin{tabular}{|c|c|c|c|c|c|c|c|}
\hline \multirow{3}{*}{ Tratamento } & \multicolumn{2}{|c|}{$\begin{array}{l}\text { Pré-incubação } \\
\text { (0 semana) }\end{array}$} & \multicolumn{4}{|c|}{$\begin{array}{c}\text { Pós-incubação } \\
\text { (2 semanas) }\end{array}$} & \multirow{3}{*}{$\begin{array}{c}\text { Pi acumulado } \\
\mathrm{mg} \mathrm{kg}^{-1}\end{array}$} \\
\hline & Po & $\mathbf{P i}$ & Po & $\Delta \mathrm{Po}_{0}$ & $\mathbf{P i}$ & $\Delta \mathbf{P}$ & \\
\hline & & $\mathrm{mg} \mathrm{kg}^{-1}$ & & $\%$ & $\mathrm{mg} \mathrm{kg}^{-1}$ & $\%$ & \\
\hline Farinha de carne e osso & 558,84 a & 124,33 a & $248,82 \mathrm{a}$ & $-55,48$ & $243,90 \mathrm{a}$ & 96,17 & $1.144,50 \mathrm{a}$ \\
\hline Lixo domiciliar & $117,02 \mathrm{~b}$ & $19,92 \mathrm{c}$ & $63,22 \mathrm{~b}$ & $-45,98$ & $35,65 \mathrm{c}$ & 79,00 & $160,26 \mathrm{c}$ \\
\hline Torta de filtro & $82,08 \mathrm{c}$ & $13,62 \mathrm{c}$ & $57,85 \mathrm{~b}$ & $-34,88$ & $25,37 \mathrm{~d}$ & 86,25 & $113,28 \mathrm{~d}$ \\
\hline Lodo de esgoto & $86,71 \mathrm{c}$ & $23,78 \mathrm{~b}$ & $35,49 \mathrm{c}$ & $-59,07$ & $43,33 \mathrm{~b}$ & 82,19 & $205,95 \mathrm{~b}$ \\
\hline Solo & $31,36 \mathrm{~d}$ & $2,01 \mathrm{~d}$ & $18,46 \mathrm{~d}$ & $-41,14$ & $2,57 \mathrm{e}$ & 28,00 & $12,65 \mathrm{e}$ \\
\hline Média & 175,20 & 36,73 & 84,77 & $-46,29$ & 70,16 & 74,32 & 327,33 \\
\hline
\end{tabular}

Pi: fósforo inorgânico; Po: fósforo orgânico; DPo: mudança percentual em relação ao tempo 0 no fósforo orgânico; e DPi: mudança percentual em relação ao tempo 0 no fósforo inorgânico. Nas colunas, médias seguidas pela mesma letra não diferem entre si pelo teste de Tukey $(\mathrm{a}=0,05)$.

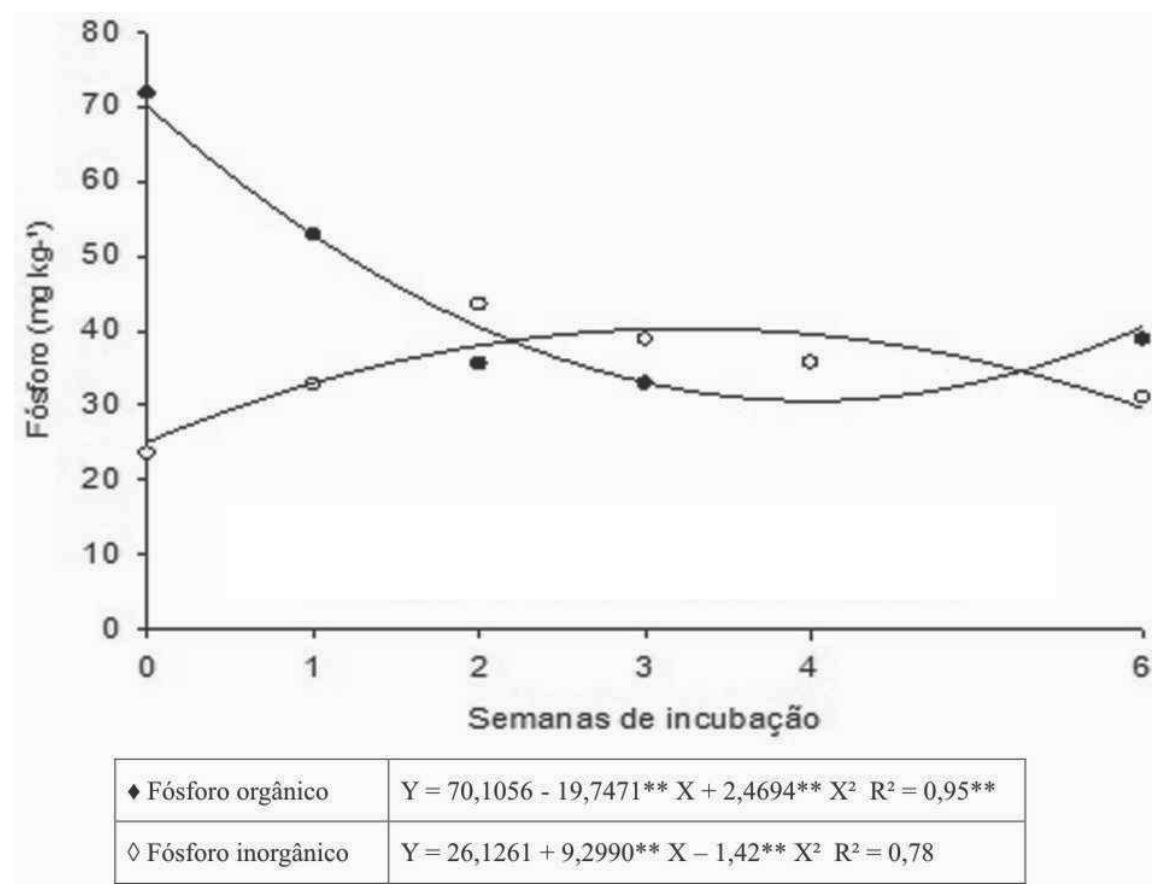

Figura 4. Teores de fósforo inorgânico (Pi) e orgânico (Po) do tratamento com composto de lodo de esgoto durante o período de incubação.

de $175,20 \mathrm{mg} \mathrm{kg}^{-1}$; o tratamento com a farinha de carne e osso apresentou o maior teor, e a torta de filtro, o menor. Para o mesmo período esses mesmos tratamentos apresentaram o maior e menor teor de P inorgânico. Após duas semanas de incubação, houve maior mudança percentual na mineralização do $\mathrm{P}$ orgânico para os tratamentos com lodo de esgoto e farinha de carne e osso (Tabela 7), refletindo os teores acumulados de $\mathrm{P}$ inorgânico ao final do período de incubação.

Na dinâmica de mineralização do $\mathrm{P}$, a forma predominante foi a orgânica, exceto para o tratamento com lodo de esgoto, no qual houve predominância das formas inorgânicas sobre as orgânicas no período compreendido entre a segunda e a quinta semanas de incubação (Figura 4). Isso indica maior presença de formas de fácil minera- lização de P nesse material, provavelmente polifosfatos incorporados aos lodos de esgoto via detergentes (Oliveira et al., 1995).

A fração do P orgânico do lodo de esgoto inicialmente diminuiu (primeiras duas semanas), incrementando gradualmente após a terceira semana de incubação. Isso pode ser explicado pela ausência do dreno-planta. Na presença de $\mathrm{P}$ inorgânico no meio, as fosfatases ácidas param de atuar (Marschner, 1995) e os microrganismos imobilizam P, incrementando a fração orgânica.

Ao final do período de incubação o tratamento com farinha de carne e osso apresentou o maior teor de $\mathrm{P}$ inorgânico, diferenciando-se dos outros tratamentos (Figura 5). Isso se deve à riqueza em $\mathrm{P}$ na farinha de carne $\mathrm{e}$ osso, com alto potencial de mineralização de formas lábeis 


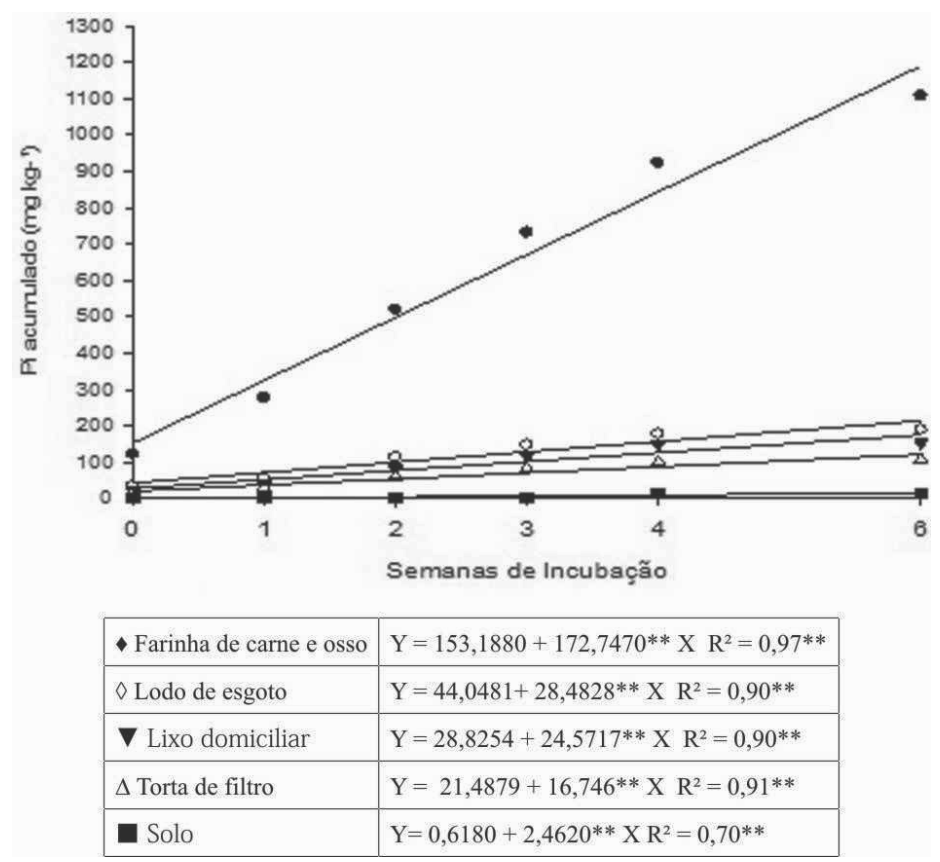

Figura 5. Teores acumulados de $\mathrm{P}$ inorgânico ( $\mathrm{Pi}$ ) para o solo tratado com resíduos e testemunha (solo) durante o período de incubação.

de P. O modelo linear simples foi ajustado aos teores acumulados de $\mathrm{P}$ inorgânico observados, estimando-se maior taxa de mineralização para o tratamento com farinha de carne e osso (178 $\left.\mathrm{m} \mathrm{kg}^{-1}\right)$, Tabela 8 . Os teores observados de $P$ inorgânico acumulados correlacionaram-se positivamente com os teores iniciais de $\mathrm{P}$ orgânico $\left(\mathrm{r}=0,99^{* *}\right)$ e negativamente com a relação $\mathrm{C} / \mathrm{P}\left(\mathrm{r}=-0,88^{* *}\right)$ dos resíduos.

\section{CONCLUSÕES}

As farinhas de osso e de carne e osso apresentaram os maiores teores totais de $\mathrm{N}, \mathrm{Pe} \mathrm{Ca}$, com relação $\mathrm{Ca} / \mathrm{P}$ da ordem de 2:1.

Os compostos de lodo de esgoto e lixo domiciliar apresentaram as menores relações $\mathrm{C} / \mathrm{N} / \mathrm{P} / \mathrm{S}$ e os maiores teores de micronutrientes.

A dinâmica de mineralização de $\mathrm{N}$ não foi explicada pela relação $\mathrm{C} / \mathrm{N}$ e pelo teor inicial de $\mathrm{N}$ nos resíduos, mas pela capacidade dos polifenóis totais solúveis em complexar proteína.

Os resíduos torta de filtro e farinha de carne e osso apresentam maior potencial de imobilização de $\mathrm{N}$ no curto prazo. Já os compostos de lixo domiciliar e lodo de esgoto apresentam o maior potencial de mineralização de $\mathrm{N}$.

Na dinâmica de mineralização das fontes orgânicas, o teor de polifenóis e sua capacidade em complexar proteína, o teor inicial de $\mathrm{N}$ total e o teor de $\mathrm{N}$ nas frações ácidos fúlvicos e húmicos influenciaram o processo de mineralização.
O teor acumulado de $\mathrm{P}$ inorgânico associou-se com o teor de $\mathrm{P}$ orgânico e com a relação C/P das fontes orgânicas. Na dinâmica de mineralização do $\mathrm{P}$, apenas a fonte orgânica lodo de esgoto apresenta predominância de formas inorgânicas sobre as orgânicas.

\section{REFERÊNCIAS}

Aggelides SM \& Londra PA (2000) Effects of compost produced from town wastes and sewage sludge on the physical properties of a loamy and clay soil. Bioresource Technology, 71:253-259.

Anderson JM \& Ingram JS (1989) Tropical soil biology and fertility: a handbook of methods. Wallingford: CAB Internacional, $171 \mathrm{p}$.

Anderson JP (1982) Soil respiration. In: Page AL, Miller RH \& Keeney D. Methods of soil analysis. Part 2. Chemical and microbiological properties. 2 ed. Madison, Soil Science Society of America. ASA. p.831-845.

Bartlett RJ \& Ross DN (1988) Colorimetric determination on of oxidizable carbon in acid soil solutions. Soil Science Society America Journal, 52:1191-1192.

Brasil (2004) Inspeção e fiscalização da produção e do comércio de fertilizantes, corretivos, inoculantes, estimulantes ou biofertilizantes, destinados à agricultura. Brasília, Ministério da Agricultura. Secretaria de Fiscalização Agropecuária, Decreto No. 4.954. 45p.

Brink RH, Dubach P \& Lynch DL (1960) Measurement of carbohydrates in soil hydrolyzates with anthrone. Soil Science, 89:157-166

CETESB (1999) Aplicação de lodos de sistemas de tratamento biológico em áreas agrícolas: critérios para projeto e operação. São Paulo, CETESB. 98p.

Deydier E, Guilet R, Sarda S, Sharrock P (2005) Physical and chemical characterisation of crude meat and bone meal combustion residue: "waste or raw material?". Journal Hazardous Materials, 121:141-148. 
Freitas SS, Cardoso CO, Camargo OA \& Lopes ES (1988) Mineralização e imobilização de nitrogênio em solo tratado com torta de filtro e carbonato de cálcio. Revista Brasileira de Ciência do Solo, 12:243-248.

Gonçalves JLM, Vaz LMS, Amaral TM \& Poggiani F (2000) Aplicabilidade de biossólido em plantações florestais: II Efeito na fertilidade do solo, nutrição e crescimento das árvores. In: Bettiol W, Bettiol W \& Camargo OA Impacto ambiental do uso do lodo de esgoto. São Paulo, EMBRAPA. p.179-196.

Hayes MHB, Maccarthy P \& Malcolm RL (1989) The search for structure: setting the scene. In: Hayes MHB Humic substances II. New York, John Wiley and Sons. p.3-31.

Hedley MJ, Stewart JWB \& Chauhan, B.S (1982) Changes in inorganic and organic soil phosphorus fractions induced by cultivation practices and by laboratory incubations. Soil Science Society of American Journal, 46:970-976.

Jorge JÁ, Camargo OA \& Valadares JMAS (1991) Condições físicas de um Latossolo Vermelho-Escuro quatro anos após aplicação de lodo de esgoto e calcário. Revista Brasileira de Ciência do Solo, 15:237-240.

Kabata PA \& Pendias H (2000) Trace elements in soils and plants. Flórida, CRC Press. 331 p.

Kempers AJ \& Zweers A (1986) Ammonium determination in soi extracts by the salicylate method. Commun. Soil Plant Analysis Sciense, 17:715-723

Kiehl EJ (1985) Fertilizantes orgânicos. Piracicaba, Editora Agronômica Ceres. p.407-459.

Kiehl EJ (2005) Adubação orgânica: 500 perguntas \& respostas. Piracicaba, Degaspari. 234p.

Malavolta E (1981) Manual de química agrícola: Adubação e adubos. $3^{a}$ ed. São Paulo, Editora Agronômica Ceres. 594p.

Marschner H (1995) Mineral nutrition of higher plants. $2^{\mathrm{a}}$ ed. San Diego, Academic Press. 889p.

Melo WJ \& Marques MO (2000) Potencial do lodo de esgoto como fonte de nutrientes para as plantas. In: Bettiol W \& Camargo OA (Eds) Impacto ambiental do uso agrícola do lodo de esgoto. Jaguariúna, EMBRAPA. p.109-141.

Morgan PH, Mercer LP \& Flodin N. (1975) General model for nutritional responses of higher organisms. Proceedings of the National Academy of Sciences, 72: 4327-4331.

Murphy J \& Riley JP (1962) A modified single solution method for determination of phosphate in natural waters. Analytica Chimica Acta, 27:31-36.
Oliveira FC, Marques MO, Bellingieri PA \& Perecin D (1995) Lodos de esgoto como fonte de macronutrientes para a cultura do sorgo granífero. Scientia Agricola, 52:360-367.

Oliveira MW, Trivelin PC, Penatt CP \& Piccolo MC (1999) Decomposição e liberação de nutrientes da palha de cana de açúcar em campo. Pesquisa Agropecuária Brasileira, 32:2359-2362.

Paul EA \& Clark FE (1996) Dynamics of residue decomposition and soil organic matter turnover. In: Soil microbiology and biochemistry. 2nd ed. San Diego, Academic Press. p.158-179.

Ribeiro Junior, JI (2001) Análises estatísticas no SAEG. Viçosa, UFV. 30p.

Rocha GN, Gonçalves JL \& Moura IM (2004) Mudanças da fertilidade do solo e crescimento de um povoamento de fertilizado com biossólido. Revista Brasileira Ciência do Solo, 28:623-639.

Rostagno HS, Albino LFT \& Donzele JL (2000) Tabelas brasileiras para aves e suínos: composição de alimentos e exigências nutricionais. Viçosa, UFV. 141p.

SAS Institute. SAS - Statistical Analysis System. SAS Institute, Inc. v. 4.10 for Windows. 1998. USA.

Siqueira JO (1988) Biotecnologia do solo: Fundamentos e perspectivas. Lavras, ABEAS. 236p.

Stevenson FJ \& Cole MA (1999) Cycles of soil: carbon, nitrogen, phosphorus, sulfur, micronutrients. New York, John Willey \& Sons. p.66-68

Tedesco MJ, Selbach PA, Gianello C \& Camargo FA (1999) Resíduos orgânicos no solo e os impactos no ambiente. In: Santos, G de A, Camargo, FA Fundamentos da matéria orgânica do solo: ecossistemas tropicais e subtropicais. Porto Alegre, Gênesis. p.159-192.

Vaz LMS \& Gonçalves JLM (2002) Uso de biossólidos em povoamento de Eucalyptus grandis: Efeito em atributos químicos do solo, no crescimento e na absorção de nutrientes. Revista Brasileira de Ciência do Solo, 26:747-758.

Xin TH, Traina SJ \& Logan TJ (1992) Chemical properties of municipal solid waste compost. Journal Environmental Quality, 21:318-329.

Yang JE, Skogley EO, Schaff BE \& Kim JJ (1998) A simple spectrometric determination of nitrate in water, resin, and soil extracts. Soil Science Society of American Journal, 62:11081115 .

Yeomans JC \& Bremner JM (1988) A rapid and precise method for routine determination of organic carbon in soil. Communication Soil Science Plant Analysis, 19:1467-1476. 